\title{
The Epidemiology of Severe Acute Respiratory Syndrome: A Global Perspective
}

\section{Evans}

\section{CH A P T ER}

\author{
Introduction \\ Epidemiological features \\ Clinical features
}

1 Incubation, infectivity and
4 transmission

8 Conclusion
10

15

\section{Introduction}

Severe acute respiratory syndrome (SARS) is a newly emerged disease caused by a previously unknown coronavirus [1-3]. It joins a long list of emerging infections [4]. However, unlike other contenders such as avian influenza, Nipah virus, Hendra virus or hantaviruses it has established the capacity for efficient human-to-human transmission and thus poses a major threat to international public health. For this reason, the World Health Organisation (WHO) has described SARS as the first serious and readily transmissible disease to emerge in the 21st century [5].

\section{Key points}

\section{SARS}

- Caused by a previously unknown coronavirus.

- Established efficient human-to-human transmission.

- First serious readily transmissible disease in 21 st century.

The first known cases of SARS occurred in Guangdong Province in southern China in late November 2002. The first official report of an outbreak of atypical pneumonia in the province on 11 February 2003 indicated that the disease had affected 305 persons and caused five deaths, and that around $30 \%$ of cases had occurred in health care workers [6]. On 21 February 2003, a medical doctor infected with SARS travelled from Guangzhou, the provincial capital, and stayed one night at a hotel in Hong Kong. He infected at least 16 other guests and visitors in the hotel. Within days, the disease began spreading around the world along international air travel routes as hotel contacts seeded hospital outbreaks in Hong Kong, Vietnam, Singapore and Canada (Figure 1.1) [7]. Hospital staff, unaware that this was a new, highly infectious disease, exposed themselves to the infection without barrier protection. Subsequently, chains of secondary transmission occurred outside the hospital environment.

These initial outbreaks were characterised by rapid increases in the numbers of cases, especially in health care workers and their close contacts (Figures 1.2 and $1.3 \mathrm{~A}, \mathrm{~B}$ ), and prompted the WHO to issue on 12 March a global alert on atypical pneumonia [8]. By 15 March 2003, the WHO had received reports of more than 150 cases of this new disease, which it named Severe Acute Respiratory Syndrome, from several Asian countries, Canada and Germany. The organisation immediately issued emergency travel 


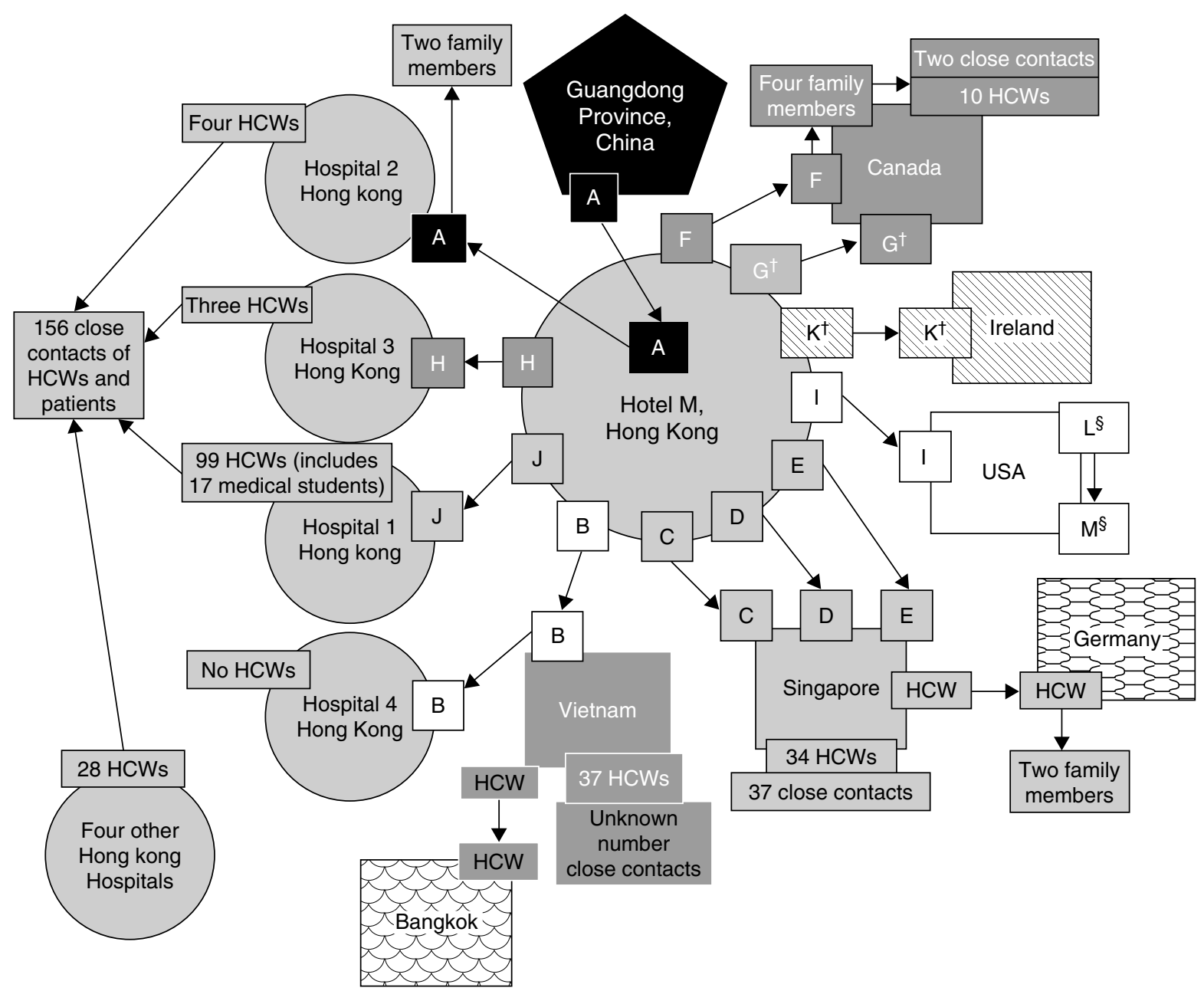

Fig. 1.1 Chain of transmission among guests at Hotel M, Hong Kong, 2003. Source: Ref. [7]. HCWs: health care workers. $\dagger$ : All guests except $G$ and K stayed on the ninth floor of the hotel. Guest $G$ stayed on the 14th floor and guest $K$ stayed on the 11 th floor. §: Guests $L$ and $M$ (spouses) were not at Hotel $M$ during the same time as index guest $A$, but were at the hotel during the same time as guests $G, H$ and $I$, who were ill during that period.

recommendations to alert health authorities, doctors and the travelling public to what was now perceived to be a worldwide threat to health [9].

The diseases spread rapidly, initially in Hong Kong [10], Hanoi [11], Toronto [12] and Singapore [13], and subsequently in the Mainland China and Taiwan [14]. Cumulative number of SARS cases passed 4000 on 23 April, 5000 on 28 April, 6000 on 2 May, 7000 on 8 May and 8000 on 28 May 2003 [15] (Figure 1.4). At the peak of the outbreak, during the beginning of May, more than 200 new cases were being reported each day.
The response to the outbreak was extraordinary. The causative agent was conclusively identified on 17 April following work through global and regional networks of virologists. On 28 April, Vietnam became the first country to stop local transmission of SARS, followed by the Philippines on 20 May and Singapore on 31 May [15]. By June, the number of new cases was gradually dwindling and by the end of June the global SARS epidemic, at least in its initial phase, was under control. There had been over 8000 cases in 30 countries worldwide and over 800 deaths (Table 1.1). 


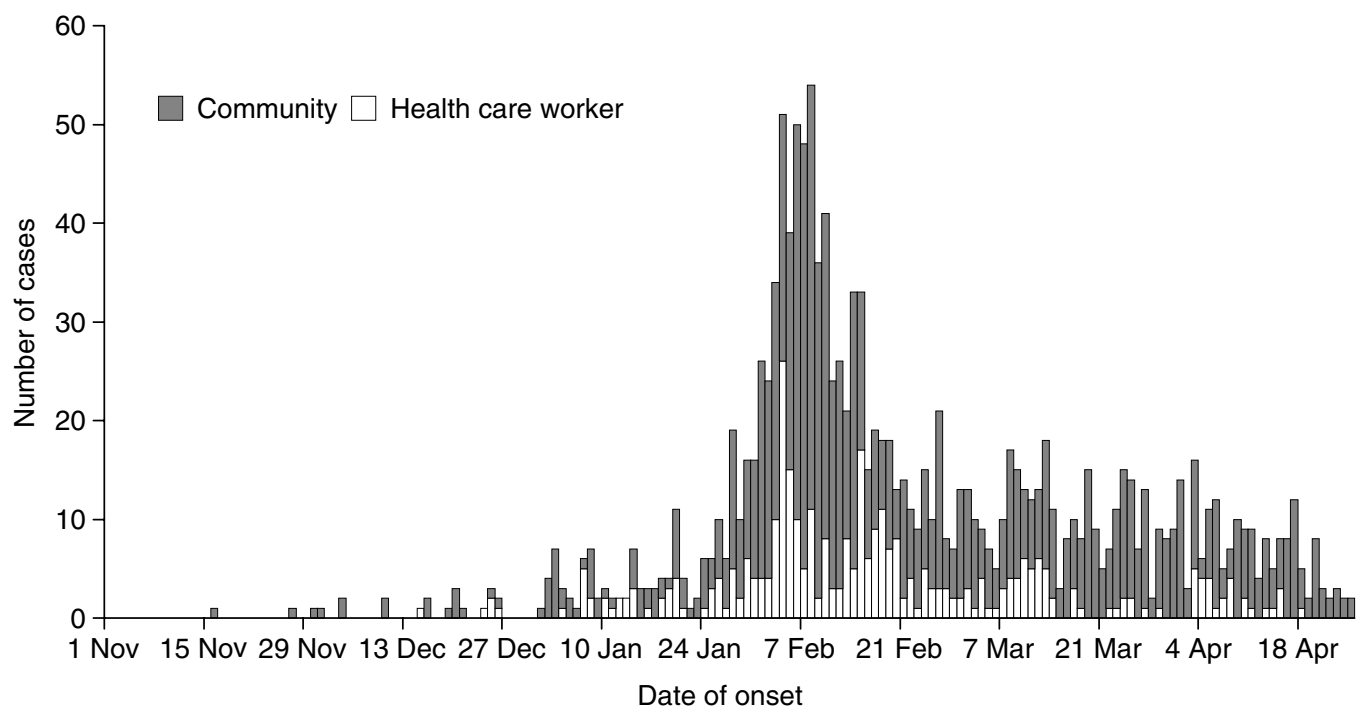

Fig. 1.2 Probable cases of SARS by date of onset in Guangdong Province, China, 1 November 2002 to 30 April $2003(n=1454)$.

\section{Case definition}

WHO has developed a clinical case definition for SARS in order to describe the epidemiology of the disease, to monitor its spread and to provide the basis for advice on prevention and control [16].

\section{Suspect case}

1. A person presenting after 1 November 2002 with history of:

- high fever $\left(>38^{\circ} \mathrm{C}\right)$

and

- cough or breathing difficulty and one or more of the following exposures during the 10 days prior to onset of symptoms:

- close contact with a person who is a suspect or probable case of SARS;

- history of travel, to an area with recent local transmission of SARS;

- residing in an area with recent local transmission of SARS.

2. A person with an unexplained acute respiratory illness resulting in death after 1 November 2002, but on whom no autopsy has been performed and

one or more of the following exposures during 10 days prior to onset of symptoms:

- close contact with a person who is a suspect or probable case of SARS;

- history of travel to an area with recent local transmission of SARS;

- residing in an area with recent local transmission of SARS.

\section{Probable case}

1. A suspect case with radiographical evidence of infiltrates consistent with pneumonia or respiratory distress syndrome (RDS) on chest X-ray (CXR).

2. A suspect case of SARS that is positive for SARS coronavirus by one or more assays.

3. A suspect case with autopsy findings consistent with the pathology of RDS without an identifiable cause.

Close contact is defined as having cared for, lived with or had direct contact with respiratory secretions or body fluids of a suspect or probable case of SARS.

\section{Exclusion criteria}

A case should be excluded if an alternative diagnosis can fully explain their illness (but not simply on the basis of a negative test for SARS coronavirus). 

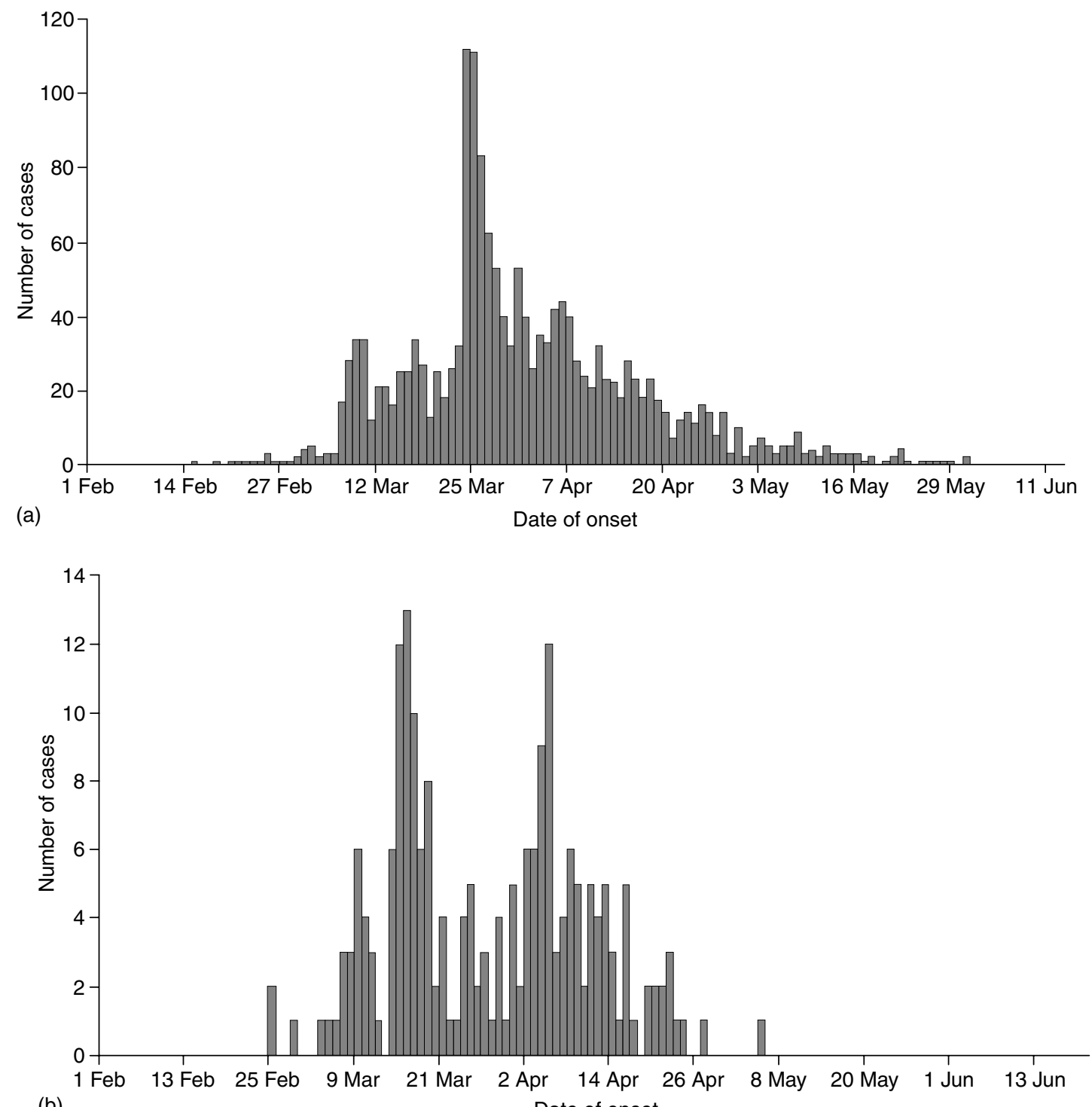

(b)

Date of onset

Fig. 1.3A Probable cases of SARS by date of onset. (a) Hong Kong SAR, China, 1 February to 16 June 2003 ( $n=1731$; as of 16 June 2003, an additional 24 probable cases of SARS have been reported from Hong Kong SAR, China, for whom no dates of onset are currently available). Source: Department of Health, Hong Kong Special Administrative Region of China. (b) Singapore, 1 February to 16 June $2003(n=206)$. Source: Ministry of Health, Singapore, WHO.

\section{Epidemiological features}

SARS is predominantly a disease of health care workers and younger adults (Table 1.2). There is a slight excess of cases in women, probably due to the predominance of female health workers. Most cases have been in people aged between 25 and 44 years.
The median age of cases was 35 years in Guangdong Province [17], 36 years in Singapore [18], 39 years in Hong Kong [10] and 45 years in Canada [19]. There have been very few cases in children and in older people. However, the highest age-specific incidence is in older people and the relatively small numbers in this age group most likely reflect the 

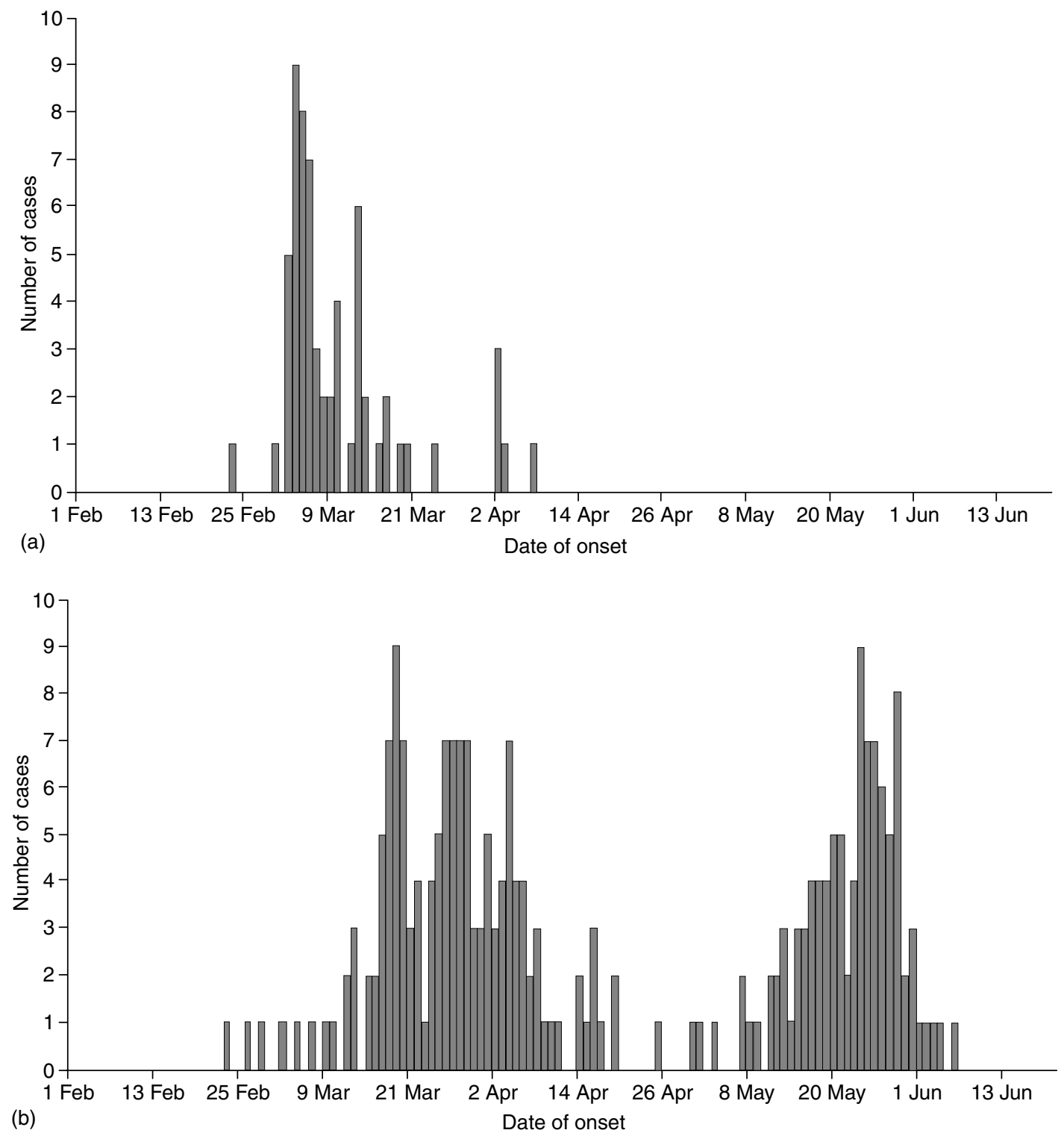

Fig. 1.3B Probable cases of SARS by date of onset. (a) Vietnam, 1 February to 16 June 2003 ( $n=62$; as of 16 June 2003 an additional probable case of SARS has been reported from Vietnam for whom no date of onset is currently available). Source: Ministry of Health, Vietnam, WHO. (b) Canada, 1 February to 13 June 2003 ( $n=242$; as of 16 June 2003, one additional probable case of SARS has been reported from Canada for whom no date of onset is available). Source: Health Canada.

younger population profile of countries such as China (Figure 1.5). This means that in countries with a substantial proportion of older people, such as many Western countries, far more cases might be expected in older people if community transmission of SARS occurs. The proportion of cases with underlying disease is between $10 \%$ and $25 \%$ depending on the setting in which infection occurs, being higher in situations where nosocomial transmission to other patients occurred as in Canada [19]. The commonest co-morbidity is diabetes or chronic heart disease. 

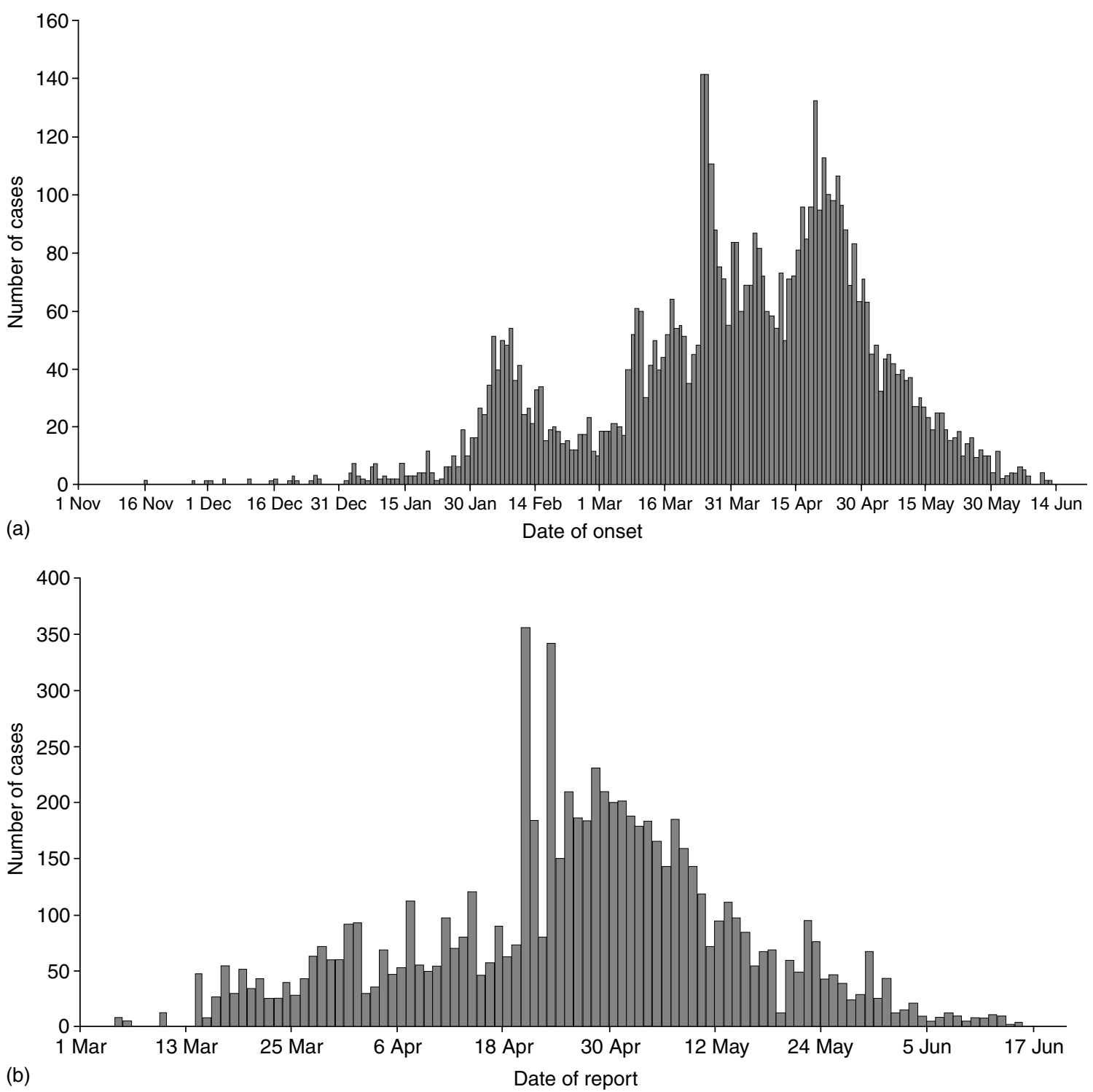

Fig. 1.4 Probable cases of SARS by date of onset, worldwide (a) 1 November 2002 to 16 June 2003 ( $n=5923$; this graph does not include 2537 probable cases (2522 from Beiijng), for whom no dates of onset are currently available). Source: Ministry of Health, China, WHO. (b) 1 March to 16 June 2003 ( $n=7563$; as of 16 June 2003, 8460 probable cases of SARS have been reported to WHO). This graph includes all cases from Hong Kong SAR, Macao SAR and Taiwan, China, but only those cases elsewhere in China reported after 3 April 2003 (1 190 cases between 16 November 2002 and 3 April 2003 not shown). Also includes 293 probable cases of SARS who have been discarded and for whom dates of report could not be identified. The USA began reporting probable cases of SARS to WHO on 20 April 2003.

Between $24 \%$ and $62 \%$ of SARS cases have been in health care workers, the proportion varying by the type of case series and declining as experience in the application of hospital infection control measures has improved [17-20]. For example, in Guangdong, the proportion of cases in health care workers was $32 \%$ in January 2003, declining to $27 \%$ in February and $17 \%$ by March and April [17] (Figure 1.1). 
Table 1.1 Cumulative number of reported probable cases of SARS and deaths, 1 November 2002 to 30 June 2003.

\begin{tabular}{lccl}
\hline Country & $\begin{array}{l}\text { Cumulative number } \\
\text { of cases }\end{array}$ & $\begin{array}{l}\text { Number of } \\
\text { deaths }\end{array}$ & $\begin{array}{l}\text { Date last probable case } \\
\text { reported (2003) }\end{array}$ \\
\hline Canada & 252 & 37 & 25 June \\
China, Mainland & 5327 & 348 & 25 June \\
China, Hong Kong SAR & 1755 & 298 & 11 June \\
China, Macao SAR & 1 & 0 & 21 May \\
China, Taiwan & 678 & 84 & 19 June \\
Europe & 34 & 0 & 4 June \\
Philippines & 14 & 2 & 15 May \\
Singapore & 206 & 32 & 18 May \\
Thailand & 9 & 2 & 7 June \\
USA & 73 & 0 & 23 June \\
Vietnam & 63 & 5 & 14 April \\
Other Asia & 23 & 2 & 31 May \\
Other & 12 & 1 & 9 June \\
Total & 8449 & 811 & 25 June \\
\hline
\end{tabular}

SAR: Special Administrative Region. Source: WHO.

Table 1.2 Case series describing epidemiological and clinical features (at presentation) of SARS.

\begin{tabular}{llllll}
\hline & $\begin{array}{l}\text { Hong Kong } \\
\text { (to 25 March) } \\
n=138 \text { [20] }\end{array}$ & $\begin{array}{l}\text { Hong Kong } \\
\text { (to 28 April) } \\
n=1425[21]\end{array}$ & $\begin{array}{l}\text { Canada } \\
\text { (to 10 April) } \\
n=144[19]\end{array}$ & $\begin{array}{l}\text { Singapore } \\
\text { (to 30 April) } \\
n=201 \text { [18] }\end{array}$ & $\begin{array}{l}\text { Guangdong } \\
\text { (to 30 April) }\end{array}$ \\
& $n=1454$ [17]
\end{tabular}


Table 1.2 (continued)

\begin{tabular}{llllll}
\hline & $\begin{array}{l}\text { Hong Kong } \\
\text { (to 25 March) } \\
n=138[20]\end{array}$ & $\begin{array}{l}\text { Hong Kong } \\
\text { (to 28 April) } \\
n=1425[21]\end{array}$ & $\begin{array}{l}\text { Canada } \\
\text { (to 10 April) } \\
n=144[19]\end{array}$ & $\begin{array}{l}\text { Singapore } \\
\text { (to 30 April) } \\
n=201 \text { [18] }\end{array}$ & $\begin{array}{l}\text { Guangdong } \\
\text { (to 30 April) }\end{array}$ \\
& $n=1454$ [17]
\end{tabular}

HCW: health care workers. a: mid-likelihood estimate and 95\% confidence intervals. b: inter-quartile range.

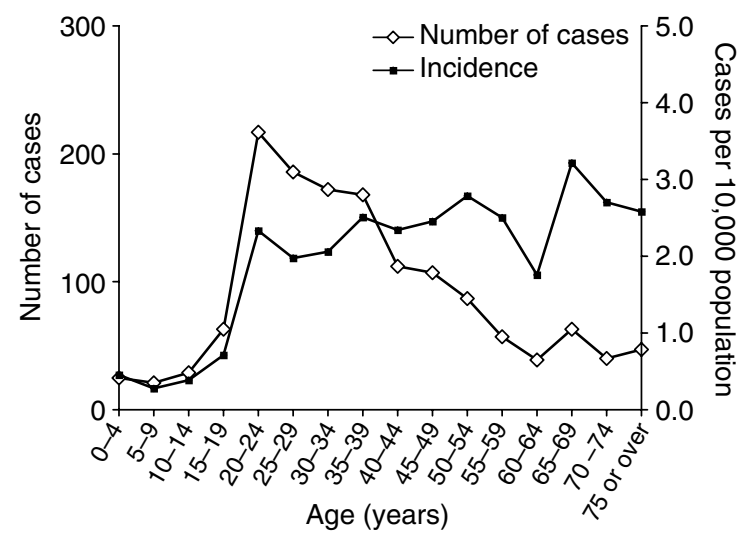

Fig. 1.5 Number of SARS cases by age and agespecific incidence, Guangdong Province, China, 1 November 2002 to 30 April 2003 ( $n=1454)$.

The largest staff group affected has been nurses, but cases have also occurred in doctors, paramedics, housekeeping staff and medical students. No cases have been reported in laboratory or pathology staff.

\section{Key points}

\section{SARS: epidemiology}

- Predominantly a disease of health care workers and young adults.

- Commonest age group 25-44 years.
- Highest age-specific incidence in older people.

- Therefore, in countries with higher proportion of older people (as in Western countries), there will be more cases in older people if community transmission of SARS occurs.

- Proportion of cases with underlying disease 10-25\%.

- Commonest co-morbidity: diabetes and chronic heart disease.

- $24-62 \%$ of SARS cases have been found in health care workers, particularly involving nurses.

\section{Clinical features}

SARS generally begins with a prodrome of fever $\left(>38^{\circ} \mathrm{C}\right)$, which is often high, often associated with chills and rigors and sometimes accompanied by other symptoms including headache, malaise and myalgia (Table 1.2). Some cases have mild respiratory symptoms though these are not prominent in the early stage of the illness. A few patients have reported diarrhoea during the febrile prodrome. After 3-7 days, a lower respiratory phase begins with the onset of a dry, non-productive cough or dyspnoea. Symptoms are milder in children $[17,21]$ while in older people respiratory symptoms may be more prominent [17]. 


\section{Key points}

\section{SARS: clinical features}

- Prodrome of fever $>38^{\circ} \mathrm{C}$.

- Chills, rigors.

- Headache, malaise, myalgia.

- Lower respiratory phase 3-7 days later. - Dyspnoea, non-productive cough.

- Symptoms milder in children.

- Respiratory symptoms more prominent in older people.

CXRs may be normal during the febrile prodrome and in about $20-25 \%$ of patients at the time of presentation. Most patients have early focal infiltrates progressing to more generalised, patchy, interstitial infiltrates. These changes are unilateral in around $40-45 \%$ of patients at presentation and bilateral in around 30\%. Some CXRs from patients in the late stages of SARS have also shown areas of consolidation.

\section{Key points}

\section{SARS: CXR}

- Normal during febrile prodrome in 20-25\%.

- Early focal infiltrates progressing to patchy interstitial infiltrates.

- Unilateral in $40-45 \%$ at presentation, bilateral in $30 \%$.

Early in the course of disease, the absolute lymphocyte count is often decreased. Over half of patients have lymphopaenia $(<1000 / \mu \mathrm{L})$ at presentation

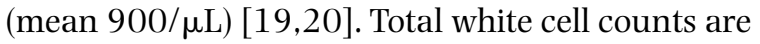
normal or decreased (mean $5 \times 10^{9} / \mathrm{L}$ ), but between $15 \%$ and $35 \%$ have leucopaenia $\left(<3.5 \times 10^{9} / \mathrm{L}\right)$. At the peak of the respiratory illness, up to half of patients have leucopaenia and thrombocytopaenia or low-normal platelet counts $(50,000-150,000 / \mu \mathrm{L})$. Early in the respiratory phase, elevated creatinine phosphokinase (CPK) levels (up to $3000 \mathrm{IU} / \mathrm{L}$ ) and hepatic transaminases (2- to 6-times the upper limits of normal) have been noted. Renal function has remained normal in the majority of patients.

\section{Key points}

SARS: laboratory tests

- $50 \%$ have lymphopaenia $(<1000 / \mu \mathrm{L})$ at presentation.

- 15-35\% have leucopaenia (<3.5 × 10\%/L) at presentation.

- At peak of illness $50 \%$ have leucopaenia and thrombocytopaenia $(50,000-$ $150,000 / \mu \mathrm{L})$.

- In respiratory phase: $\uparrow$ CPK (>3000 IU/L) and hepatic transmission (two to six liver normal).

- Normal renal function tests in majority.

In $10-20 \%$ of cases, the respiratory illness is severe enough to require intubation and mechanical ventilation. The overall case fatality among persons with SARS is estimated to be $14-15 \%$, but ranges from $0 \%$ to $50 \%$ depending on age. Case fatality is less than $1 \%$ in persons aged 24 years or younger, $6 \%$ in persons aged $25-44$ years, $15 \%$ in persons aged 45-64 years and more than $50 \%$ in persons aged 65 years and older $[21,22,23]$. Patients with underlying disease, such as diabetes, are more likely to have a poor outcome [19].

\section{Key points}

\section{SARS: clinical features}

- $10-20 \%$ require intubation/mechanical ventilation.

- Overall case fatality 14-15\% (range: 0-50\%).

- Case fatality:

- $<1 \%$ in patients aged $<24$ years;

$-6 \%$ in patients between 25 and 44 years of age;

- $15 \%$ in patients between 45 and 64 years of age;

- $>50 \%$ in patients aged $\geqslant 65$ years.

- Poorer outcome in patients with underlying disease. 


\section{Incubation, infectivity and transmission \\ Incubation period}

Knowing the incubation period of a disease, which is the time from exposure to a causative agent to onset of symptoms, is particularly important as it can help clinicians in making the diagnosis and it forms the basis for many recommended control measures, such as contact tracing and the duration of home isolation of contacts. The incubation period for SARS - based on analysis by WHO of individuals with well-defined single-point exposures in Singapore, Canada and Europe - is usually 2-7 days but may be as long as 10 days [23]. The incubation period will vary from one case to another according to the route by which the person was exposed, the dose of virus received and other factors such as immune status. Although there have been anecdotal reports of incubation periods longer than 10 days, these have not been corroborated.

\section{Period of infectivity}

The initial rapid spread of SARS in hospitals in Hanoi, Vietnam and in Hong Kong first indicated that the disease was highly contagious. Since then it has become clear that most cases of SARS occur in close contacts of patients, particularly household members, and in health care workers, other patients and visitors inadvertently exposed to a case $[10,12]$. The period of infectivity appears to be greatest after the person with SARS develops respiratory symptoms, but transmission may occur during the prodromal period [24] (Figure 1.6). Persons with SARS do not appear to be infectious during the incubation period or after febrile symptoms have resolved. Further viral studies will help clarify the stages of the illness when virus shedding is greatest and the concentration of virus in various body fluids.

\section{Key points}

Incubation period: 2-7 days (may be as long as 10 days).

Period of infectivity:

- greatest after patient develops respiratory symptoms;

- may occur during prodrome;

- not during incubation period;

- not after afebrile symptoms have resolved.

\section{Routes of transmission}

SARS is spread in the majority of cases through close contact with an infected person. Transmission occurs mainly through exposure to infected large or medium droplets expelled during coughing or sneezing, and

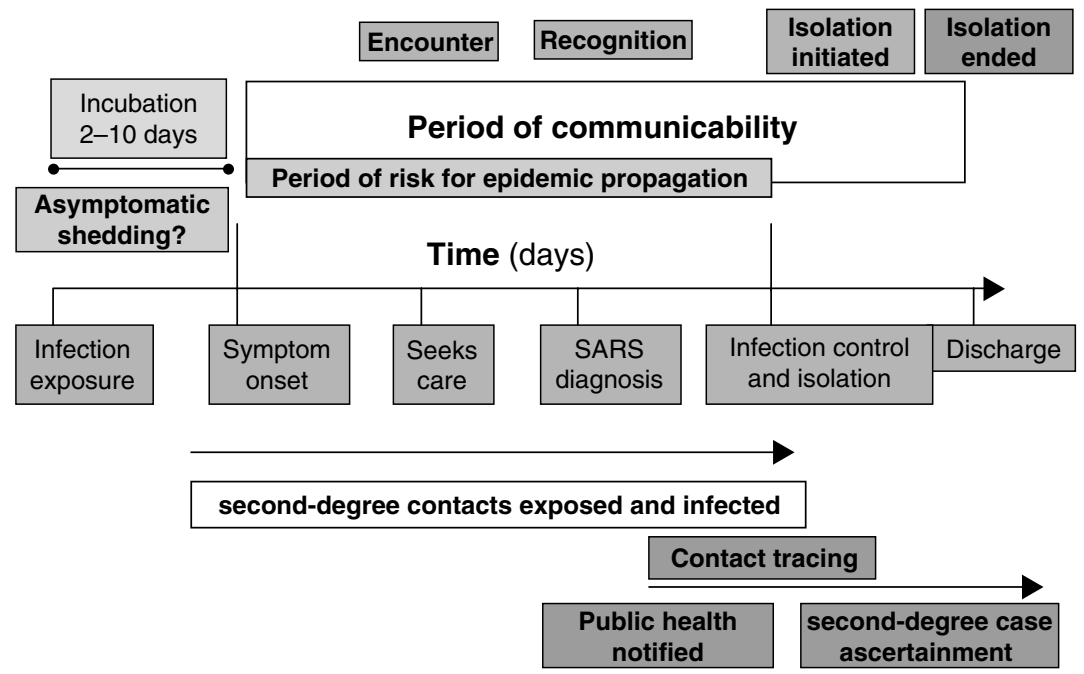

Fig. 1.6 A contagion model for SARS. 
probably also occasionally from contact with contaminated fomites $[10,12]$. Likelihood of contagion seems to be similar to that described with other viral respiratory tract pathogens, such as respiratory syncytial virus and rhinovirus [25]. Shedding of the SARS virus in respiratory secretions, faeces and urine is now well established [24,26]. The role of body fluids (such as saliva, tears and urine) and of faeces in transmission of infection is less clear. In vitro laboratory tests show that the virus can survive in faeces for at least 2 days, and in urine for at least 24 hours [26]. Virus in faeces taken from patients suffering from diarrhoea, which has a lower acidity than normal stools, can survive for 4 days raising the possibility that surfaces contaminated by diarrhoea could be an important source of infection. However, the dose of virus needed to cause infection remains unknown and further studies are needed before the role of faecal-oral transmission can be determined.

\section{Key points}

Routes of transmission

- Close contact with infected person.

- Droplets expelled during coughing/ sneezing.

- Contact with contaminated fomites.

\section{Transmission in hospital}

Health care workers are at high risk of SARS. Most infections have occurred either before infection control procedures were instituted or where procedures have not been properly followed. Observations from Hong Kong suggest that this risk is greatest when a hospital receives its first admissions of SARS, when patients are admitted to a general ward, and when large numbers of patients are admitted over a short period of time [27]. Nosocomial spread is less likely when patients are admitted directly to a designated ward. One case-control study of risk factors for transmission in hospital staff caring for patients with SARS found that fewer staff who wore masks and gowns, and washed their hands became infected compared with those who did not, though only not wearing a mask was independently associated with increased risk [28]. Investigation of one incident among protected health care workers suggested that ill-fitting masks or contamination during mask removal were factors in infection [29].

\section{Key points}

SARS: transmission in hospital

Factors involved:

- infection control measures;

- admission/cohorting procedures in wards;

- use of gowns, masks and hand washing.

\section{Transmission in the community}

SARS transmits readily within the household setting, although if patients are isolated early in the course of the illness, secondary transmission to other members of the household can be prevented [30]. Other instances of spread in the community are rare with the highest risk probably being in those exposed to symptomatic patients in confined areas such as taxi drivers, and airline staff and passengers. A review by WHO of 35 flights with symptomatic probable SARS cases on board identified four flights during which in-flight transmission may have occurred [31]. One flight was associated with 22 secondary cases including two flight attendants. The affected passengers were seated within seven rows in front and five rows behind the index case suggesting that the infection spread through exposure to respiratory droplets. Cases in other settings are rare though there have been reports of transmission in the banqueting room of a restaurant, in the workplace and at a wholesale market [18].

In contrast to experience in hospitals, there have been very few large outbreaks in the community. One notable exception was a large and sudden cluster of over 300 cases that occurred in residents of the Amoy Gardens housing estate in Hong Kong [32]. Cases associated with this cluster were much more likely to present with diarrhoea and to require intensive care compared with previously documented SARS cases. This raised the possibility of transmission by a different route and infection with a high virus load, such as might happen following exposure to a concentrated environmental source. Subsequent investigations ruled out airborne or 
waterborne transmissions and suggested that the outbreak was primarily caused by contaminated sewage entering households through dried U-traps in the bathroom floor drain.

\section{Key points}

SARS: transmission in community

- Transmits readily in household setting.

- Early isolation of patients prevents secondary transmission to other household members.

- Reports of transmission in taxis, aircraft.

\section{Superspreaders/superspreading event patient}

On average, one SARS generates around three secondary cases [30]. However, certain individuals with SARS who have been implicated in spreading the disease to numerous (10 or more) other individuals, and these cases have been described as 'superspreaders' [18,33] (Figure 1.7). Several such individuals have been described particularly during the early days of the SARS outbreak. They include the hotel index case in Hong Kong who triggered the worldwide dissemination of SARS as well as the index case for each of the outbreaks in Vietnam and Singapore $[7,11,13]$. Superspreaders have also been described with other diseases, such as Ebola [34].

The explanation for the apparent high infectivity of these patients remains unknown. It may be that they were co-infected with another virus and that this caused them to secrete an exceptionally high amount of infectious material or that some other factor, perhaps in the environment, amplified the potential for transmission at some key phase of virus shedding. Alternatively, high secondary attack rates in hospital staff, relatives and other visitors that some superspreaders caused may be a consequence

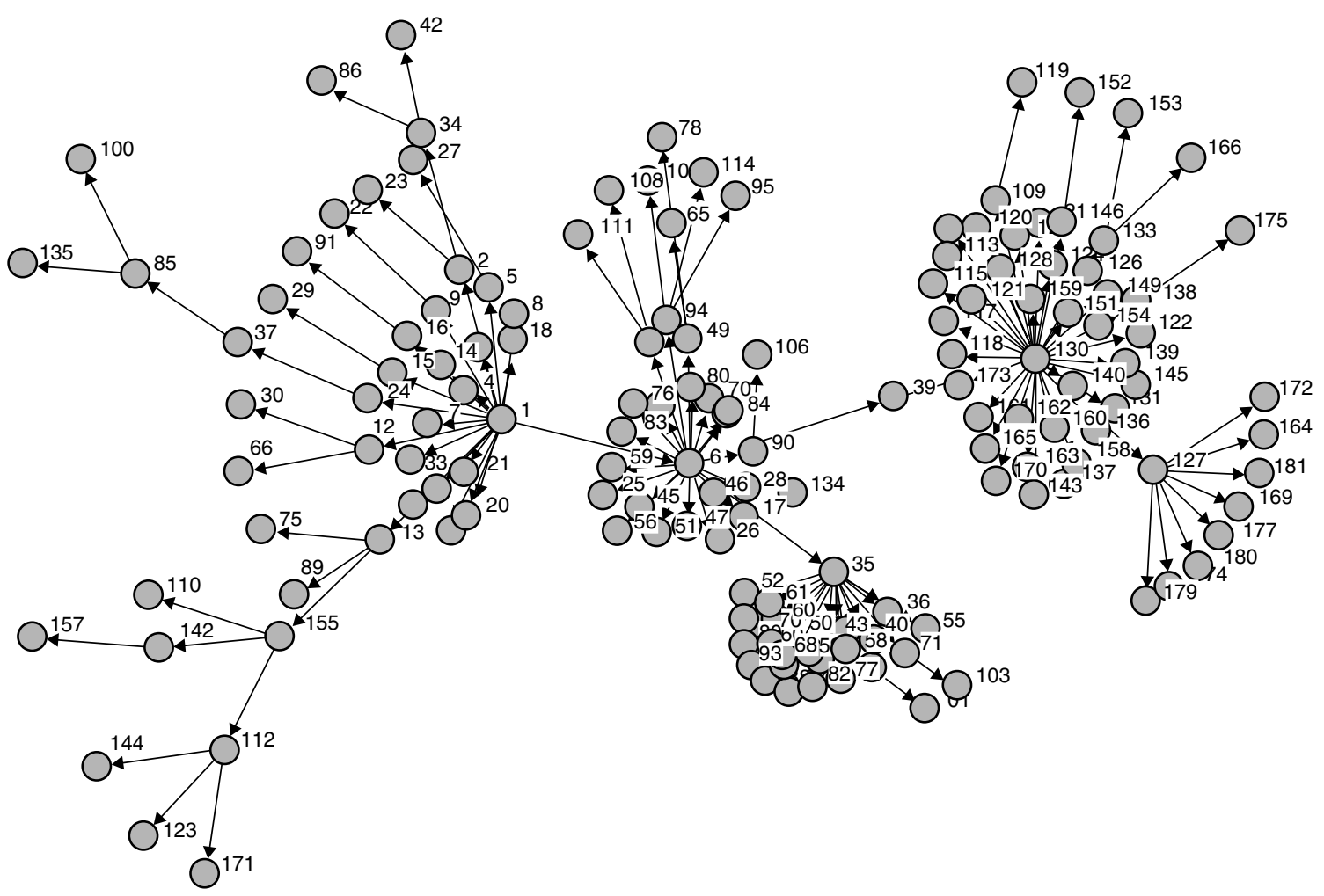

Fig. 1.7 Probable cases of SARS by reported source of infection showing superspreaders (cases 1, 6, 35, 127 and 130), Singapore, 25 February to 30 April 2003. Source: Morb Mortal Wkly Rep 2003; 52: 405-41 1. 
of the absence of stringent hospital infection control measures before the infectivity of SARS was recognised. Isolation of contacts, rapid admission of cases to hospital and improved hospital infection control have all been shown to significantly reduce the average number of new cases of SARS arising from a single-source case [30].

\section{Prevention and control}

\section{Breaking the chain of transmission}

At present, the most effective way to control SARS is to break the chain of transmission from infected to healthy people. There are three key actions necessary to achieve this: early case detection, patient isolation and contact tracing (Table 1.3) [35].

Case detection aims to identify a SARS case as soon as possible after the onset of symptoms. Once a case is identified, the next step is to ensure they are isolated promptly and managed according to strict infection control procedures. Finally, it is vital to identify all close contacts of each case and make sure that they are carefully followed up, including daily health checks and voluntary home isolation.

These measures limit the daily number of contacts possible for each potentially infectious case. By shortening the amount of time that elapses between onset of illness and isolation of the patient the opportunity for the virus to spread to others is reduced, as is the average number of new cases generated by each case (the effective reproduction number) [21,30] (Figure 1.6). If each new SARS patient infects, on average, less than one person then the outbreak will die out, otherwise the disease will continue to spread.

Table 1.3 Detection and protection measures to control SARS.

- Prompt identification of persons with SARS, their movements and contacts

- Effective isolation of SARS patients in hospitals

- Appropriate protection of health care workers treating SARS patients

- Comprehensive identification and isolation of suspected SARS cases

- Exit screening of international travellers

- Timely and accurate reporting and sharing of information with other authorities and/or governments

\section{Surveillance and reporting}

Good surveillance and rapid reporting is the basis of case detection and is required to instigate contact tracing and promptly identify outbreaks. Most surveillance systems rely on notification of infectious diseases by clinical practitioners. In the case of SARS, this has been achieved by voluntary reporting by clinicians of cases meeting the WHO case definition firstly to local public health authorities and then onwards to national ministries of health and the WHO itself. The use of electronic systems can assist in the rapid reporting and response to suspect cases.

The current clinical case definition has worked well to control the SARS outbreak but a more precise definition will be needed for longer-term surveillance [36]. Until a highly reliable, sensitive and specific early test is available, diagnosis of SARS will inevitably depend on evaluation of symptoms and history of contact. When local transmission of SARS has ceased, clusters of atypical pneumonia and nosocomial transmission to health care staff or hospital visitors will become important sentinel events for recognising new instances of SARS.

\section{Isolation of patients}

The earliest possible isolation of all suspect and probable cases of SARS in hospital is vital. A short time between the onset of symptoms and isolation of the patient reduces opportunities for transmission of infection to other people and reduces the number of contacts that require active follow-up [30]. It also gives patients the best chance of receiving lifesaving care, should their condition take a critical course. In an outbreak of SARS every effort should be made to reduce the average time from the onset of symptoms to isolation under 3 days.

\section{Key points}

SARS: prevention and control

Breaking the chain of transmission:

- Early case detection/surveillance and reporting of cases.

- Patient isolation.

- Contact tracing. 


\section{Hospital infection control}

Health care settings seem to provide an ideal setting for outbreaks of SARS, and have served to amplify and propagate the infection. The reasons are unclear but appear to be a combination of factors such as the potential for aerosol-generating procedures, poor ventilation and air flow patterns, and the presence of vulnerable patients. Inadequate training or compliance with infection control, high workload and cryptic clinical presentations can compound the problem. Meticulous infection control procedures are necessary to prevent nosocomial spread including [37]:

- Comprehensive triage arrangements in emergency departments and clinics.

- Adequate facilities for patient isolation with appropriate air flow control.

- Scrupulous hand hygiene.

- Use of appropriate personal protective equipment.

- Avoidance of aerosol-generating procedures.

SARS has clearly demonstrated that a single case admitted to an unprepared hospital can ignite a new outbreak.

The infection control measures for a radiology department are discussed in a separate chapter towards the end of the book.

\section{Contact tracing and isolation of contacts}

The case should be interviewed by a trained health care worker as soon as possible after the diagnosis of SARS is made, either face-to-face or by telephone. If the patient is too ill to be interviewed a proxy contact history should be obtained from the nextof-kin. The date of onset of symptoms should be corroborated and details of all close contacts since that date obtained. Close contacts are defined as anyone who cared for, lived with or had direct contact with respiratory secretions or body fluids or stool of person with SARS [16].

All close contacts should be followed up for 10 days from the last date of contact with the case. Quarantine or home confinement of contacts was employed with considerable success to bring the outbreaks in the Mainland China, Hong Kong and Singapore under control. The temperature of all contacts should be monitored daily, preferably by a health worker. It may be necessary to make arrangements to provide the household with food supplies and other essential commodities during the period of observation. In overcrowded households it may be preferable to relocate the contacts to alternative accommodation to limit the risk of secondary transmission within the household.

If the source of infection in the index case is not known, a full contact history for the 10 days before the date of onset of symptoms should be obtained. This will include details of all persons who may have had face-to-face contact with the case, as well as information on the workplace, visits to hospitals or other health care facilities, visits to crowded places, details of journeys on public transport, and overnight stays outside the home. Where there are several cases with an unidentified source, it is important to correlate contact histories to identify any common links.

\section{Key points}

\section{Contact tracing}

- The case (if possible) or next-of-kin should be interviewed by trained health care workers.

- Date of onset of symptoms should be corroborated.

- Details of all contacts must be obtained.

- Contacts should be followed up for 10 days from last date of contact with the case.

- The temperature of all contacts should be monitored daily.

- Quarantine of contacts may be necessary.

\section{Management of an outbreak}

Each hospital, and public health services at each level within the health system, should have an outbreak plan that can be used to guide the response to a SARS incident. It should include the following:

- clear aims and objectives;

- a description of the kind of circumstances in which an outbreak should be declared and an Outbreak Control Team (OCT) convened;

- the terms of reference for the OCT;

- the proposed membership of the OCT and an outline of the roles and responsibilities of individual members; 
- arrangements for planning and implementing appropriate control measures, and for securing urgent additional resources, if required;

- details of lines of communication with partner organisations and agencies, central government, health professionals, the general public and the media;

- arrangements for declaring the outbreak over and preparing a final written report.

A final report of the outbreak should be prepared and presented to the responsible authority each time an OCT is convened. The plan should regularly updated and revised in the light of changing circumstances.

\section{Public education}

An outbreak of a serious infectious disease that is poorly understood and for which no treatment is available inevitably produces great public concern. As far as possible, it is important to give the public clear and unambiguous information about the nature of the disease and the risk it poses. Mass-media campaigns to educate the public were an important part of the response to SARS. The wearing of a mask, other than by those in close contact with a suspect or probable case, is unlikely to be of value. However, information about the symptoms of the disease and advice to encourage prompt reporting of symptoms is sensible. In some circumstances, the establishment of fever clinics to screen individuals with symptoms and to relieve pressure on emergency rooms may be helpful [36].

\section{Border control measures and travel precautions}

In areas where person-to-person transmission of SARS has been documented, screening of travellers and border control measures are indicated [38,39]. All travellers should be made aware of the symptoms of SARS and advised to seek immediate medical attention should symptoms occur. Departing international passengers should be asked to complete a brief questionnaire on any history of contact with a suspect or probable SARS case, or any symptoms of SARS during the previous 48 hours, and be screened for fever by means of a temperature check. Travellers with fever should be requested to postpone travel and to seek medical attention. Persons meeting the SARS case definition should be referred to a health care facility.
Table 1.4 In-flight care of a suspected case of SARS.

- Isolate passenger, as far as possible, from other passengers and crew

- Provide passenger with protective mask

- Identify toilet for exclusive use of the ill passenger

- Carer(s) should wear protective mask and gloves and wash hands after contact with the ill passenger

- Captain should radio ahead to alert port health authorities at the destination airport

- Identify all contacts on board (household members, flight attendants and passengers in the same row or two rows in front or behind)

- On arrival, place passenger in isolation until assessed by port health authorities

If a passenger on a flight from a SARS-affected area becomes noticeably ill with a fever and respiratory symptoms, the cabin crew are recommended to take basic precautions (Table 1.4) [39]. If medical assessment concludes that the person is a suspect or probable case of SARS all contacts during the flight should be identified and followed up in accordance with the WHO guidance.

\section{Conclusion}

The speed with which the SARS epidemic was managed demonstrates the decisive power of high-level political commitment to contain an outbreak. Extensive use of measures like patient isolation, contact tracing and follow-up, quarantine, public education and travel precautions have worked to bring the disease under control even in the absence of effective treatments or vaccines [40].

Many unanswered questions about SARS remain, particularly about the origin of the coronavirus. If the disease has, as suspected, a wild animal reservoir, then it may become endemic at low levels or re-emerge as a seasonal or epidemic infection. SARS has features that can thwart even the best plans. The initial symptoms may masquerade as many other diseases, the incubation period is long enough to allow it to spread to any part of the globe without detection, and one single highly infectious case may set off a chain of transmission leading to a hundred or more additional cases. Continued worldwide vigilance, particularly for any hospital-based cluster of febrile patients or health care workers with respiratory symptoms, will therefore be needed for some time to come [15]. 


\section{References}

1. Peiris JS, Lai ST, Poon LL et al. Coronavirus as a possible cause of severe acute respiratory syndrome. Lancet 2003; 361: 1319-1325.

2. Ksiazek TG, Erdman D, Goldsmith CS et al. A novel coronavirus associated with severe acute respiratory syndrome New Engl J Med 2003; 348: 1953-1966.

3. Drosten C, Gunther S, Preiser W et al. Identification of a novel coronavirus in patients with severe acute respiratory syndrome. New Engl J Med 2003; 348: 1967-1976.

4. Morse SS. Factors in the emergence of infectious diseases. Emerg Infect Dis 1995; 1: 7-15.

5. World Health Organisation. Severe Acute Respiratory Syndrome (SARS): Status of the Outbreak and Lessons for the Immediate Future. Geneva, World Health Organisation, 20 May 2003. Available at: http://www.who.int/csr/media/sars_wha.pdf (accessed 28 May 2003).

6. Rosling L and Rosling M. Pneumonia causes panic in Guangdong province. Br Med J 2003; 326: 416.

7. Centers for Disease Control and Prevention. Outbreak of severe acute respiratory syndrome - worldwide, 2003. Morb Mortal Wkly Rep 2003; 52: 241-248.

8. World Health Organisation. WHO Issues Global Alert about Cases of Atypical Pneumonia: Cases of Severe Respiratory Illness May Spread to Hospital Staff. Geneva: World Health Organisation, 12 March 2003. Available at: http://www.who.int/ medicentre/releases/2003/pr22/en/ (accessed 14 May 2003).

9. World Health Organisation. Severe Acute Respiratory Syndrome Available at: http://www.who.int/csr/sarsarchive/2003_ 03_15/en/ (accessed 14 May 2003).

10. Tsang KW, Ho PL, Ooi GC et al. A cluster of cases of severe acute respiratory syndrome in Hong Kong. New Engl J Med 2003; 348: 1977-1985.

11. Vu TH, Cabau JF, Nguyen NT and Lenoir M. SARS in North Vietnam. New Engl J Med 2003; 348: 2035.

12. Poutanen SM, Low DE, Henry B et al. Identification of severe acute respiratory syndrome in Canada. New Engl J Med 2003; 348: 1995-2005.

13. Hsu L-Y, Lee C-C, Green JA et al. Severe acute respiratory syndrome (SARS) in Singapore: clinical features of index patient and initial contacts. Emerg Infect Dis 2003; 6: 713-717.

14. Twu S-J, Chen T-J, Chen C-J et al. Control measures for severe acute respiratory syndrome (SARS) in Taiwan. Emerg Infect Dis 2003; 6: 718-720.

15. World Health Organisation. Severe acute respiratory syndrome (SARS): over 100 days into the outbreak. Wkly Epidemiol Rec 2003; 78: 217-220.

16. World Health Organisation. Case Definitions for Surveillance of Severe Acute Respiratory Syndrome (SARS). Available at: http://www.who.int/csr/sars/casedefinition/en/ (accessed 16 May 2003).

17. Xu R-H, He J-F, Evans MR et al. Epidemiologic clues to the origin of severe acute respiratory syndrome in China (personal communication).

18. World Health Organisation. Severe acute respiratory syndrome - Singapore 2003. Wkly Epidemiol Rec 2003; 78: $157-162$

19. Booth CM, Matukas LM, Tomlinson GA et al. Clinical features and short-term outcomes of 144 patients with SARS in the Greater Toronto area. I Am Med Assoc 2003; 289: 1-9.

20. Lee N, Hui D, Wu A et al. A major outbreak of severe acute respiratory syndrome in Hong Kong. New Engl J Med 2003; 348: 1986-1994.

21. Donnelly C, Ghani AC, Leung GM et al. Epidemiological determinants of spread of causal agent of severe acute respiratory syndrome in Hong Kong. Lancet 2003; 361: 1761-1766.

22. Hon KLE, Leung CW and Cheng WFT. Clinical presentations and outcome of severe acute respiratory syndrome in children. Lancet 2003: 361: 1701-1703.
23. World Health Organisation. Update 49. SARS Case Fatality Ratio, Incubation Period. Available at: http://www.who.int/ csr/sarsarchive/2003_05_07a/en/ (accessed 14 May 2003).

24. Peiris JSM, Chu CM, Cheng VCC et al. Clinical progression and viral load in a community outbreak of coronavirusassociated SARS pneumonia: a prospective study. Lancet 2003; 361: 1767-1772.

25. Musher DM. How contagious are common respiratory infections? New Engl J Med 2003; 348: 1256-1266.

26. World Health Organisation. Update 47. Studies of SARS Virus Survival, Situation in China. Available at: http://www. who. int/csr/sarsarchive/2003_05_05/en/ (accessed 14 May 2003).

27. Chan-Yeung M, Seto WH, Sung JJ et al. Severe acute respiratory syndrome: patients were epidemiologically linked. $\mathrm{Br}$ Med J 2003; 326: 1393.

28. Seto WH, Tsang D, Yung RW et al. Effectiveness of precautions against droplets and contact in prevention of nosocomial transmission of severe acute respiratory syndrome (SARS). Lancet 2003; 361: 1519-1520.

29. Centers for Disease Control and Prevention. Cluster of severe acute respiratory syndrome cases in protected health care workers - Toronto, Canada, April 2003. Morb Mortal Wkly Rep 2003; 52: 433-436.

30. Riley S, Fraser C, Donnelly CA et al. Transmission dynamics of the etiological agent of SARS in Hong Kong: impact of public health interventions. Science 2003; 300: 1961-1966.

31. World Health Organisation. Update 62. More Than 8000 SARS Cases Reported Globally, Situation in Taiwan, Data on In-Flight Transmission, Report on Henan Province, China. Available at: http://www.who.int/csr/sarsarchive/2003 05_22/en/ (accessed 23 May 2003).

32. Hong Kong Department of Health. Main Findings of an Investigation into the Outbreak of Severe Acute Respiratory Syndrome at Amoy Gardens. Available at: http://www.info. gov.hk/dh/ap.htm (accessed on 19 April 2003).

33. World Health Organisation. Update 30. Status of Diagnostic Test, Significance of Superspreaders, Situation in China. Available at: http://www.who.int/csr/sarsarchive/2003_04_15/en/ (accessed 14 May 2003).

34. Khan AS et al. The re-emergence of Ebola hemorrhagic fever, Democratic Republic of Congo, 1995. J Infect Dis 1999; 179(Suppl 1): S76-S86.

35. World Health Organisation. Update 54. Outbreaks in the Initial Hot Zones Indicate that SARS Can be Contained. Available at: http://www.who.int/csr/sarsarchive/2003_05_13/en/ (accessed 14 May 2003).

36. Rainer TH, Cameron PA, Smit D et al. Evaluation of WHO criteria for identifying patients with severe acute respiratory syndrome out of hospital: prospective observational study. Br Med J 2003; 326: 1354-1358.

37. World Health Organisation. Hospital Infection Control Guidelines for Severe Acute Respiratory Syndrome. Available at: http://www.who.int/csr/sars/infectioncontrol/en/ (accessed 19 May 2003)

38. World Health Organisation. Update 11. WHO Recommends New Measures to Prevent Travel-Related Spread of SARS. Available at: http://www.who.int/csr/sarsarchive/2003_ 03_27/en/ (accessed 14 May 2003).

39. World Health Organisation. WHO recommended measures for persons undertaking international travel from areas affected by severe acute respiratory syndrome (SARS). Wkly Epidemiol Rec 2003; 78: 97-99.

40. World Health Organisation. Vietnam SARS-free. Wkly Epidemiol Rec 2003; 78: 145-146. 